\title{
Die Therapie der schweren Sepsis
}

\section{Treatment of Severe Sepsis}

Autor

Institut

\section{J. Lorenz}

Klinik für Pneumologie, Internistische Intensivmedizin, Infektiologie und Schlafmedizin, Klinikum Lüdenscheid

\section{Bibliografie}

DOI $10.1055 / \mathrm{s}-0028-1119702$

Online-Publikation: 16. 3. 2009

Pneumologie 2009; 63:

197-204 @ Georg Thieme

Verlag KG Stuttgart · New York ISSN 0934-8387

\section{Korrespondenzadresse}

Prof. Dr. med. Joachim Lorenz

Klinik für Pneumologie, Internistische Intensivmedizin, Infektiologie und Schlafmedizin Klinikum Lüdenscheid

Paulmannshöher Straße 14

58515 Lüdenscheid joachim.lorenz@klinikumluedenscheid.de

Serienherausgeber

S. Rosseau, Berlin

B. Schönhofer, Hannover

\section{Zusammenfassung \\ $\nabla$}

Als Sepsis wird eine Infektion mit systemischer Manifestation bezeichnet. Bei Vorliegen mindestens eines Organversagens handelt es sich um eine schwere Sepsis. Die Bedeutung des Krankheitsbildes in der Pneumologie ergibt sich aus der Tatsache, dass in der nichtoperativen Medizin die Pneumonie die häufigste Sepsisursache und das akute Lungenversagen eine der Hauptmanifestationen der schweren Sepsis darstellen. Die empirische Basis der Sepsisbehandlung ist einem erheblichen Wandel unterworfen, da im letzten Jahrzehnt erstmals durch die Bildung von Studiengruppen und Kompetenzzentren die Durchführung großer, aussagekräftiger Interventionsstudien ermöglicht wurde. Neben der Kontrolle der zugrunde liegenden Infektion umfasst die Therapie der schweren Sepsis die Behandlung hämodynamischer, respiratorischer, endokrinologischer, koagulatorischer und immunologischer Aspekte der Erkrankung. Während die neuen Konzepte der Schockbehandlung und der Beatmungstherapie bestätigt werden konnten, sind Ansätze der Antikoagulation und die endokrinologische und die immunologische Therapie noch im Fluss.

\section{Abstract \\ $\nabla$}

The sepsis syndrome is defined as a clinically suspected infection accompanied by systemic manifestations. The emergence of one or multiple organ dysfunctions is the hallmark of severe sepsis. The significance of sepsis in pulmonary medicine is highlighted by the fact that severe pneumonia is the most prevalent cause of sepsis in non-surgical intensive care medicine and that acute lung injury is among the prominent manifestations of severe sepsis. The formation of major study groups and competence centres during the last decade has made meaningful prospective interventional studies possible that, in turn, have resulted in the development of clinical treatment algorithms. Today, the multidisciplinary treatment of severe sepsis includes haemodynamic, respiratory, endocrine, immune, and coagulation aspects besides the control of the underlying infection. While new concepts of shock treatment and mechanical ventilation have been established by means of confirmatory studies, endocrine interventions as well as immune and anticoagulation procedures are still under investigation.

\section{Einleitung}

\section{$\nabla$}

Definition und Schweregrade der Sepsis wurden 1992 vom „American College of Chest Pysicians“ und der „Society of Critical Care Medicine“ festgelegt [1] und seitdem wiederholt leicht modifiziert [2]. Als Sepsis wird die systemische Reaktion auf eine Infektion bezeichnet. Um sie von banalen, fieberhaften Infekten zu unterscheiden, wird von einer Sepsis gesprochen, wenn bei klinischem Verdacht einer Infektion mindestens zwei der folgenden Kriterien der systemischen Entzündungsreaktion („systemic inflammatory response syndrome“, SIRS) erfüllt sind: Fieber oder Hypother- mie, Leukozytose oder Leukozytopenie oder unreife Neutrophile im Blutbild, Tachykardie und Tachypnoe/Hypokapnie. Ein SIRS per se kommt auch bei nichtinfektiösen Erkrankungen, zum Beispiel beim Trauma, bei Verbrennung, Pankreatitis und Intoxikationen vor und hat als solches eine geringe Spezifität in der Identifikation von Risikopatienten für die Entwicklung einer schweren Sepsis oder eines septischen Schocks. In einer Europäischen Kohortenstudie betrug die kumulative Inzidenz der Progression zu schwerer Sepsis oder septischem Schock bei SIRS lediglich 20\% nach 10 und 24\% nach 30 Tagen [3]. Daher kommt dem begründeten Verdacht einer Infektionser- 


\begin{tabular}{|c|c|c|c|}
\hline $\begin{array}{l}\text { Infektion/ } \\
\text { Noxe }\end{array}$ & Sepsis & schwere Sepsis & $\begin{array}{l}\text { Abb. } 1 \text { Definition und Schweregrade der Sepsis } \\
\text { (nach 1). SIRS: Systemic inflammatory response } \\
\text { syndrome. }\end{array}$ \\
\hline $\begin{array}{l}\text { eine klinische Reaktion auf eine } \\
\text { unspezifische Noxe, die } 2 \text { oder mehr } \\
\text { der folgenden Symptome aufweist } \\
\text { - Temperatur } \geq 38^{\circ} \mathrm{C} \text { oder } \leq 36^{\circ} \mathrm{C} \\
\text { - Herzfrequenz } \geq 90 / \text { min } \\
\text { - Atemfrequenz } \geq 20 / \text { min oder } \\
\text { Hyperventilation }\left(\mathrm{PaCO}_{2}\right. \\
\leq 33 \mathrm{mmHg}) \\
\text { - Leukozyten } \geq 12000 / \mathrm{mm}^{3} \text { oder } \\
\leq 4000 / \mathrm{mm}^{3} \text { oder }>10 \% \text { unreife } \\
\text { Neutrophile }\end{array}$ & $\begin{array}{l}\text { SIRS mit } \\
\text { angenommener } \\
\text { oder nachge- } \\
\text { wiesener Infektion }\end{array}$ & $\begin{array}{l}\text { Sepsis }+1 \text { akutes } \\
\text { Organversagen } \\
\text { refraktäre Hypotension } \\
\text { (Schock) } \\
\text { Nierenversagen } \\
\text { Lungenversagen } \\
\text { Leberversagen } \\
\text { Thrombozytopenie } \\
\text { Koagulopathie (INR >1,5) } \\
\text { Enzephalopathie } \\
\text { (Verwirrtheit) } \\
\text { metabolische Azidose } \\
\text { ohne erkennbare Ursache }\end{array}$ & \\
\hline
\end{tabular}

krankung eine hohe Bedeutung zu. Es ist auch zu unterscheiden zwischen einer Bakteriämie und der Sepsis. Die Sepsis verläuft oft ohne Erregernachweis im Blut, während viele Infektionen mit positiver Blutkultur ohne schwere Sepsis verlaufen.

Eine schwere Sepsis ist durch das Vorliegen mindestens eines Organversagens im Rahmen der Sepsis gekennzeichnet. Dabei kommen die organbezogenen Definitionen der Funktionsstörungen zum Tragen. So ist das akute Lungenversagen (ARDS) gekennzeichnet durch eine akut auftretende hypoxämische Insuffizienz der Atmung mit einem Verhältnis des $\mathrm{paO}_{2}$ zum $\mathrm{F}_{\mathrm{i}} \mathrm{O}_{2}$ von $<200$, begleitet von beidseitigen Lungeninfiltraten bei fehlenden Befunden einer dekompensierten Linksherzinsuffizienz. Ein septischer Schock liegt vor bei einer Sepsis mit ansonsten nicht erklärbar erniedrigtem Blutdruck (systolisch $\leq 90 \mathrm{mmHg}$, Mitteldruck: $\leq 70 \mathrm{mmHg}$ ), der sich refraktär gegen einen adäquaten Volumenersatz verhält. Ein septisches Multiorganversagen besteht, wenn die Kriterien der akuten Organinsuffizienz für mindestens zwei Organe erfüllt sind $[1,4,5]$. $\bullet$ Abb. 1 erläutert die Definition der Sepsis und ihre Schweregrade.

Nach nordamerikanischen Zahlen betrug 1993 der Anteil der schweren Sepsis an der Gesamtaufnahmerate in Krankenhäusern $2 \%$, in Intensivstationen dagegen $59 \%$ und $25 \%$ der Sepsisfälle gingen mit einem septischen Schock einher [6]. In einer großen Beobachtungsstudie betrug die Inzidenz der Sepsis 3,0 Fälle/ 1000 Einwohner und 2,26 Fälle pro 100 Krankenhausentlassungen. Die Krankenhaussterblichkeit betrug $28,6 \%$, was zur Schätzung eines Anteils von 9,5\% an allen Todesfällen führte [7]. Eine prospektive französische Studie über 8 Jahre in 22 Kliniken ergab einen Anteil des septischen Schocks von 8,2\% an allen Krankenhausaufnahmen mit einem Anstieg von 7,0\% im Jahr 1993 auf $9,7 \%$ in 2000 [8]. Die Gesamtletalität betrug 60,1\% und fiel im Beobachtungszeitraum von $62,1 \%$ auf $55,9 \%$. Das relative Risiko zu versterben, gegenüber den Intensivpatienten ohne Sepsis, war 3,9-fach erhöht. Im Vergleich mit anderen Schockpatienten war bei septischem Schock die Sterblichkeit in der Intensivstation um $11,7 \%$ (57,1 vs. 45,4) höher und die Liegedauer in der Intensivstation um 7,0 Tage (16,8 vs. 9,8) verlängert. In einer Beobachtungsstudie des Deutschen Kompetenznetzes „SepNet“ über das Jahr 2003 in 310 Kliniken betrug die Prävalenz einer Infektion bei Intensivpatienten 34,7\%. Unter den Infizierten betrug der Anteil von Patienten mit schwerer Sepsis oder septischem Schock 30,8\%. Die Letalität der Betroffenen betrug 54\% [9].

Die Sepsis nimmt ihren Ausgang im Allgemeinen von einer Organinfektion, die meist - jedoch nicht immer - klinisch zu fassen ist. Die häufigste Quelle ist in etwa $40 \%$ der Fälle eine ambulant oder nosokomial erworbene Pneumonie [10]. Andere häufige Sepsisquellen sind Abdominalinfektionen, Weichgewebeinfektionen, Harnwegsinfektionen, ZNS-Infektionen, die Endokarditis oder Fremdkörper-/Katheter-Infektionen. Die Häufigkeit der verschiedenen Organquellen schwankt sehr zwischen verschiedenen Typen von Intensivstationen (z. B. chirurgisch vs. Internistisch). Der relative Anteil der bakteriellen Sepsis durch gramnegative Erreger ist über die Zeit gefallen (auf etwa 25\%), während grampositive und polymikrobielle Sepsisursachen auf 30-50\% der Fälle zugenommen haben. Auch die Sepsis durch multiresistente Bakterien und Pilze nimmt zu (etwa 25\% der Fälle) [5].

Eine Sepsis ist eher eine wirtstypische Verlaufsform als die Folge einer Infektion durch einen bestimmten Erreger. Daher ist die Beachtung individueller Risikofaktoren wichtig. Zu solchen Risikofaktoren gehören eine zelluläre oder humorale Immuninkompetenz, die Splenektomie und schwere chronische Erkrankungen mit Organinsuffizienz. Genetische Varianzen im angeborenen Immunsystem disponieren unabhängig davon zu schweren Verlaufsformen im Fall einer Infektion. So konnte gezeigt werden, dass spezifische Polymorphismen im Toll-like Rezeptor 1-Gen bei Sepsispatienten zu Organdysfunktion und Tod disponieren [11].

Die Diagnosestellung bewegt sich entlang der klinischen Kriterien der Sepsis und umfasst außerdem die Diagnostik der zugrundeliegenden Organinfektion. Im Hinblick auf die oben angedeuteten Limitationen der Definitionskriterien für die Sepsis [2] wurden zahlreiche Biomarker auf ihr Vermögen, zwischen SIRS nicht infektiöser Genese, nicht septischen Infektionen und der Sepsis zu unterscheiden, untersucht. Serumspiegel des Prohormons Procalcitonin sind in den letzten Jahren intensiv untersucht worden. Sie korrelieren besser mit dem Schweregrad einer Infektion als das Creaktive Protein, aber sind nicht ausreichend spezifisch, um eine Sepsis zu definieren. Das Serum-Procalcitonin ist aber zumindest aufgrund seines hohen negativen prädiktiven Wertes und der kurzen Serumhalbwertzeit dazu in der Lage, bei niedrigem Serumspiegel $(<0,25 \mathrm{ug} / \mathrm{l})$ eine bakterielle Sepsis weitgehend auszuschließen und die Beendigung einer Therapie mit Antiinfektiva zu begründen $[12,13]$. Serielle Bestimmungen des Procalcitonins haben das Potenzial zur Therapiesteuerung [14].

Die Therapie der schweren Sepsis ist notwendigerweise multidimensional und zielt sowohl auf die zugrunde liegende Infektion, wie auf die assoziierten Organdysfunktionen. In den letzten Jahrzehnten haben sich auf der Basis von Expertenerfahrungen The- 


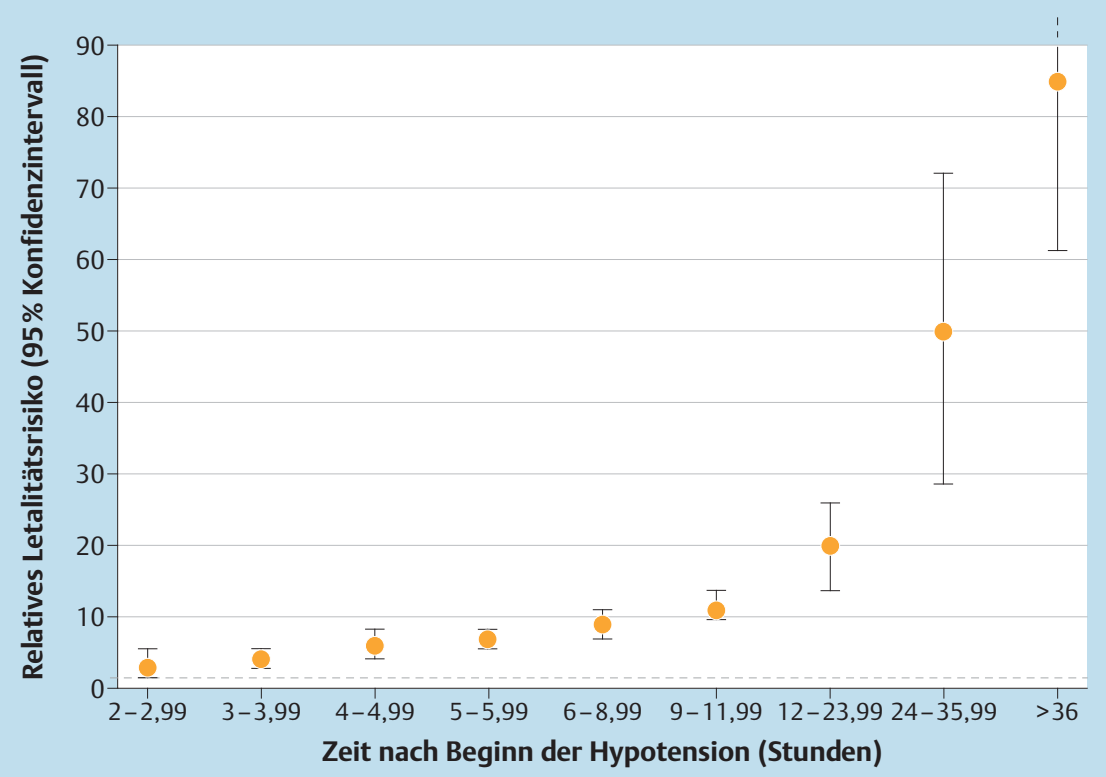

Abb. 2 Letalitätsrisiko (adjusted odds ratio, + $95 \%$ Konfidenzintervall) bei septischem Schock in Abhängigkeit vom Zeitintervall bis zur Initiierung einer angemessenen antimikrobiellen Therapie im Vergleich zur Letalität bei Therapiebeginn in der ersten Stunde des Schockes (nach: 21).

rapiemodalitäten herausgebildet, die allgemein akzeptiert waren, aber über eine schwache wissenschaftliche Evidenz verfügten. Erst in den letzten zehn Jahren hat sich die empirische Grundlage der Sepsisbehandlung erheblich verbessert und sie mündete in die Entwicklung einfacher, klinisch einsetzbarer $\mathrm{Al}$ gorithmen. Einige der grundlegenden Empfehlungen basieren jedoch auf lediglich einer oder zwei Studien, sodass sich die gesamte Sepsistherapie noch im Umbruch befindet. Überdies ist die Implementierung zentraler Komponenten der Therapie in die intensivmedizinische Praxis ein schleppender Prozess. Eine Audit-kontrollierte Befragung zu Therapiegewohnheiten durch das Deutsche Kompetenznetzwerk „SepNet“ ergab, dass zum Beispiel die maschinelle Beatmung mit kleinen Atemzugvolumina als Standard beim ARDS im Jahr 2003 in 92\% bekannt und akzeptiert war, aber nur in $4 \%$ praktiziert wurde [15].

Die folgenden Ausführungen konzentrieren sich auf empirisch gesicherte Behandlungsverfahren in den zentralen Dimensionen der Sepsisbehandlung. Ungeachtet dessen werden weitere Behandlungsformen und Medikamente geprüft, die das Potenzial haben, die Patientenbetreuung zu bereichern. Dazu gehören zum Beispiel immunologisch wirkende Substanzen wie Immunglobuline [16], monoklonale Antikörper gegen Tumornekrosefaktor-alpha $[17,18]$, gerinnungsaktive Substanzen wie der Tissue Factor [19], Selen [20] und viele andere.

\section{Infektionskontrolle}

$\nabla$

Die Kontrolle der der Sepsis zugrunde liegenden Infektion mit chirurgischen Methoden, der Entfernung infizierter Fremdkörper und der Gabe von Antiinfektiva folgt dem gesunden Menschenverstand. Eine direkte Evidenz liegt dazu aber nicht vor und es wird sie auch zukünftig nicht geben, da Placebo-kontrollierte Studien ethisch nicht vertretbar wären. Zahlreiche observationelle Kohortenstudien belegen den Nutzen der Infektionstherapie jedoch indirekt und zeigen übereinstimmend, dass die Verzögerung einer angemessenen antimikrobiellen Therapie bei dem Verdacht einer schweren Sepsis oder eines septischen Schocks schon nach der ersten Stunde nach Symptombeginn mit einer Verschlechterung der Prognose einhergeht. Eine retrospektive nordamerikanische Kohortenstudie zum septischen Schock ergab, dass mit jeder Stunde Verzögerung einer effektiven antimikrobiellen Therapie nach Beginn des Blutdruckabfalls die Überlebensrate um im Mittel 7,6\% fiel. Sie betrug bei Therapiebeginn in der ersten Stunde $79,9 \%$, in der zweiten Stunde 70,5\%, in der sechsten Stunde $42,0 \%$ und in der 10 . bis 12 . Stunde nur noch 25,4\% ( $\bullet$ Abb. 2).

Das Zeitintervall bis zum Therapiebeginn war in der multivariaten Analyse der stärkste Einzelprädiktor der Letalität [20]. Ähnliche Daten wurden publiziert für die Candidämie [22], die schwere ambulant erworbene Pneumonie [23,24], die Ventilator-assoziierte Pneumonie [25], die Legionellose [26], die bakterielle Meningitis [27] und für die Bakteriämie durch Pseudomonas aeruginosa [28] oder Staphylococcus aureus [29]. Aus diesen Befunden ergibt sich die Forderung, mit der empirischen antimikrobiellen Therapie so schnell wie möglich zu beginnen. Die erforderliche Diagnostik darf die Therapie nicht verzögern.

Auswahlkriterien für Antiinfektiva richten sich nach der vermuteten Sepsisquelle, dem Setting bei Erwerb der Infektion (ambulant, nosokomial, Intensivstation), bei nosokomialen Infektionen nach der Dauer des Aufenthaltes im Krankenhaus oder in der Intensivstation, nach dem Vorliegen von Risikofaktoren für multiresistente Erreger und nach dem Immunstatus des Patienten. Patienten mit einer definierten Immuninkompetenz (z.B. AIDS, Knochenmarksinsuffizienz, Therapie mit Immunsuppressiva) haben ein hohes Risiko, außer durch bakterielle Pathogene durch atypische Erreger (z. B. Pneumocystis jiroveci, Cytomegalievirus, opportunistische Pilze) zu erkranken. In $\bullet$ Tab. 1 ist als Beispiel die Therapieempfehlung zur schweren, ambulant erworbenen Pneumonie aus der Leitlinie der Deutschen Gesellschaft für Pneumologie und der Paul-Ehrlich-Gesellschaft für Chemotherapie [30] wiedergegeben.

In der Regel werden bei einem septischen Verlauf Breitspektrumantibiotika mit hoher Gewebepenetration intravenös eingesetzt. Die Frage der Monotherapie oder Kombinationstherapie ist nicht abschließend geklärt. Die dazu vorliegenden Studien zeigen widersprüchliche Ergebnisse [31 - 36]. Die Studie von Baddour zeigt aufschlussreich, dass bei bakteriämischer Pneumokokkeninfektion ohne schweren Verlauf kein Unterschied im Therapieergebnis zwischen einer Monotherapie und einer Kombinationsthera- 
Tab. 1 Therapieempfehlung für die kalkulierte Initialtherapie bei Patienten mit schwerer ambulant erworbener Pneumonie (sCAP) nach: Leitlinie der Deutschen Gesellschaft für Pneumologie und Beatmungsmedizin und Paul-Ehrlich-Gesellschaft für Chemotherapie [30]

\begin{tabular}{|c|c|c|}
\hline \multirow{5}{*}{$\begin{array}{l}\text { ohne Risiko einer Infektion durch } \\
\text { Pseudomonas aeruginosa }\end{array}$} & Mittel der Wahl & Alternative \\
\hline & $\begin{array}{l}\text { Breitspektrum Betalaktam-Antibiotikum } \\
\text { Ureidopenizillin+Betalaktamaseinhibitor } \\
\text { (z. B. Piperacillin . Tazobactam) }\end{array}$ & $\begin{array}{l}\text { Pneumokokkenaktives Fluorchinolon } \\
\text { Levofloxacin, Moxifloxacin }\end{array}$ \\
\hline & $\begin{array}{l}\text { Oder Cephalosporin Gruppe IIla } \\
\text { Ceftriaxon, Cefotaxim }\end{array}$ & \\
\hline & $\begin{array}{l}\text { Oder Pseudomonas-inaktives Carbapenem } \\
\text { Ertapenem }\end{array}$ & \\
\hline & $\begin{array}{l}\text { + Makrolid } \\
\text { Azithromycin, Clarithromycin }\end{array}$ & \\
\hline \multirow{5}{*}{$\begin{array}{l}\text { mit Risiko einer Infektion durch } \\
\text { Pseudomonas aeruginosa } \\
\text { (strukturelle Lungenerkrankung, } \\
\text { vorangegangener Pseudomonas- } \\
\text { Nachweis) }\end{array}$} & $\begin{array}{l}\text { Pseudomonas-aktives Betalaktam-Antibiotikum } \\
\text { Ureidopenizillin+Betalaktamaseinhibitor } \\
\text { (z. B. Piperacillin. Tazobactam) }\end{array}$ & $\begin{array}{l}\text { Pseudomonas-aktives Betalaktam Antibiotikum } \\
\text { Ureidopenizillin + Betalaktamaseinhibitor } \\
\text { (z. B. Piperacillin. Tazobactam) }\end{array}$ \\
\hline & $\begin{array}{l}\text { Oder Cephalosporin Gruppe IV } \\
\text { Cefepim }\end{array}$ & $\begin{array}{l}\text { Oder Cephalosporin Gruppe IV } \\
\text { Cefepim }\end{array}$ \\
\hline & $\begin{array}{l}\text { Oder Pseudomonas-aktives Carbapenem } \\
\text { Imipenem, Meropenem, Doripenem }\end{array}$ & $\begin{array}{l}\text { Oder Pseudomonas-aktives Carbapenem } \\
\text { Imipenem, Meropenem, Doripenem }\end{array}$ \\
\hline & $\begin{array}{l}\text { +Pseudomonas-akives Fluorchinolon } \\
\text { Levofloxacin, Moxifloxacin }\end{array}$ & $\begin{array}{l}+ \text { Aminoglykosid } \\
\text { Amikacin, Gentamycin, Tobramycin }\end{array}$ \\
\hline & & + Makrolid (s. o.) \\
\hline
\end{tabular}

pie besteht, während bei schwerer Erkrankung mit Intensivpflege (schwere Sepsis) die Kombinationstherapie eindeutig überlegen ist [36]. Im Hinblick auf die Therapiedauer liegen keine Hinweise vor, dass eine Behandlung über mehr als 8 bis 10 Tage einer kürzeren Therapie überlegen ist [37]. Ausnahmen sind Infektionen durch nicht-fermentierende, gramnegative Stäbchen (zum Beispiel Pseudomonas aeruginosa). Eine weitere Untersuchung zeigte, dass bei Vorliegen eines septischen Schocks eine Kombinationstherapie überlegen war [38]. Aktuelle Studien zeigen, dass durch die tägliche serielle Verwendung von Biomarkern die Therapiedauer invidualisiert werden kann [14]. Diese Strategie führte zu einer Verkürzung der mittleren Behandlungsdauer.

\section{Optimierung des Sauerstofftransportes}

Das septische Kreislaufversagen ist gekennzeichnet durch eine Kombination von infektiös-toxisch bedingter Gefäßdilatation und eine vasale Leckage mit Flüssigkeitsextravasation in den dritten Raum. Seltener kommt es außerdem zu einer septischen Kardiomyopathie mit Abfall der kardialen Auswurfleistung. Dagegen ist ein reaktiv bedingter, supranormaler Herzindex typisch. Als einfache diagnostische Methoden in der Beurteilung der Hämodynamik bieten sich die Echokardiographie, die Messung des zentralvenösen Druckes und der gemischt venösen (oder auch zentral venösen) Sauerstoffsättigung an. Sowohl die Verzögerung einer raschen Schockbehandlung wie der Einsatz von Vasopressoren vor dem Flüssigkeitsersatz verschlechtern die Überlebensprognose [39]. Darauf basierend schlugen Rivers u. Mitarb. 2001 einen Algorithmus zur Soforttherapie des septischen Schockes basierend auf physiologischen Variablen („early goal directed therapy“) vor. Das Konzept hat sich als praktikabel erwiesen und sein Potenzial zur Prognoseverbesserung konnte wiederholt bestätigt werden. Die Implementierung in der Notaufnahme ist möglich [40]. In der nosokomialen Sepsis wurde das Konzept bisher nicht geprüft. Wie in $\bullet$ Abb. 3 dargestellt wird, erfolgt zunächst eine Anhebung des zentral venösen Drucks auf 8-12 $\mathrm{mmHg}$ (bei maschineller Beatmung auf $12 \mathrm{mmHg}$ ) durch intensive Applikation von Volumenersatzmitteln, gefolgt von einer An- hebung des arteriellen Mitteldrucks auf mindestens $65 \mathrm{mmHg}$ durch Vasopressoren.

Reicht diese Behandlung nicht aus, eine zentral venöse Sauerstoffsättigung von mindestens $70 \%$ zu erreichen, so wird der Hämatokrit durch Gabe von Erythrozytenkonzentraten auf über $30 \%$ angehoben. Alternativ oder ergänzend können, insbesondere bei Nachweis einer nicht supranormalen linkskardialen Auswurfleistung positiv inotrop wirkende Substanzen - vorzugsweise Dobutamin [41] - eingesetzt werden. Der gemeinsame Nenner all dieser Maßnahmen ist die Verbesserung der Sauerstofftransportkapazität in das Gewebe, die mittels eines pulmonal-arteriellen Katheters aus den Parametern Sauerstoffsättigung (arteriell und zentral venös), Hämoglobin und Herzminutenvolumen auch direkt bestimmt werden kann. Das Konzept von Rivers u. Mitarb. ist mit Hilfe des Algorithmus in $\mathbf{A b b} \mathbf{3}$ aber auch ohne invasive hämodynamische Diagnostik durchführbar. Die mittels einer konventionellen Standardtherapie kontrollierte, randomisierte Untersuchung ergab zugunsten der „early goal directed therapy“ eine erniedrigte 30-Tage-Letalität von 30,5 gegenüber 46,5\% in der Kontrollgruppe. Die Organfunktionen verbesserten sich schneller und erreichten ein höheres Niveau. In der Verumgruppe wurden mehr Volumenersatzmittel, häufiger Dobutamin und mehr Erythrozytenkonzentrate verabreicht und die Therapie begann wesentlich früher.

Die Auswahl geeigneter Volumenersatzmittel und Vasopressoren war in jüngster Zeit Gegenstand empirischer Untersuchungen. Wie Metaanalysen bereits 2001 ergaben [42], ist der Volumenersatz mittels Humanalbumin obsolet, da seine Verabreichung mit einer höheren Letalität einhergeht. Kolloide Volumenersatzmittel (zum Beispiel Hydroxyäthylstärke, HAES) haben den Vorteil einer rascheren Anhebung des mittleren arteriellen und zentral venösen Druckes im Vergleich zu kristalloiden Lösungen, sind aber mit der potenziellen Gefahr einer Nierenschädigung verbunden. Eine vergleichende Wertung war bis 2008 nicht möglich [43], bis die VISEP-Studie zeigte, dass die Verabreichung von HAES im Vergleich zu Ringer-Laktat zur raschen Volumentherapie häufiger und dosisabhängig mit schweren renalen Komplikationen einherging, die die Rate schwerer unerwünschter Ereig- 


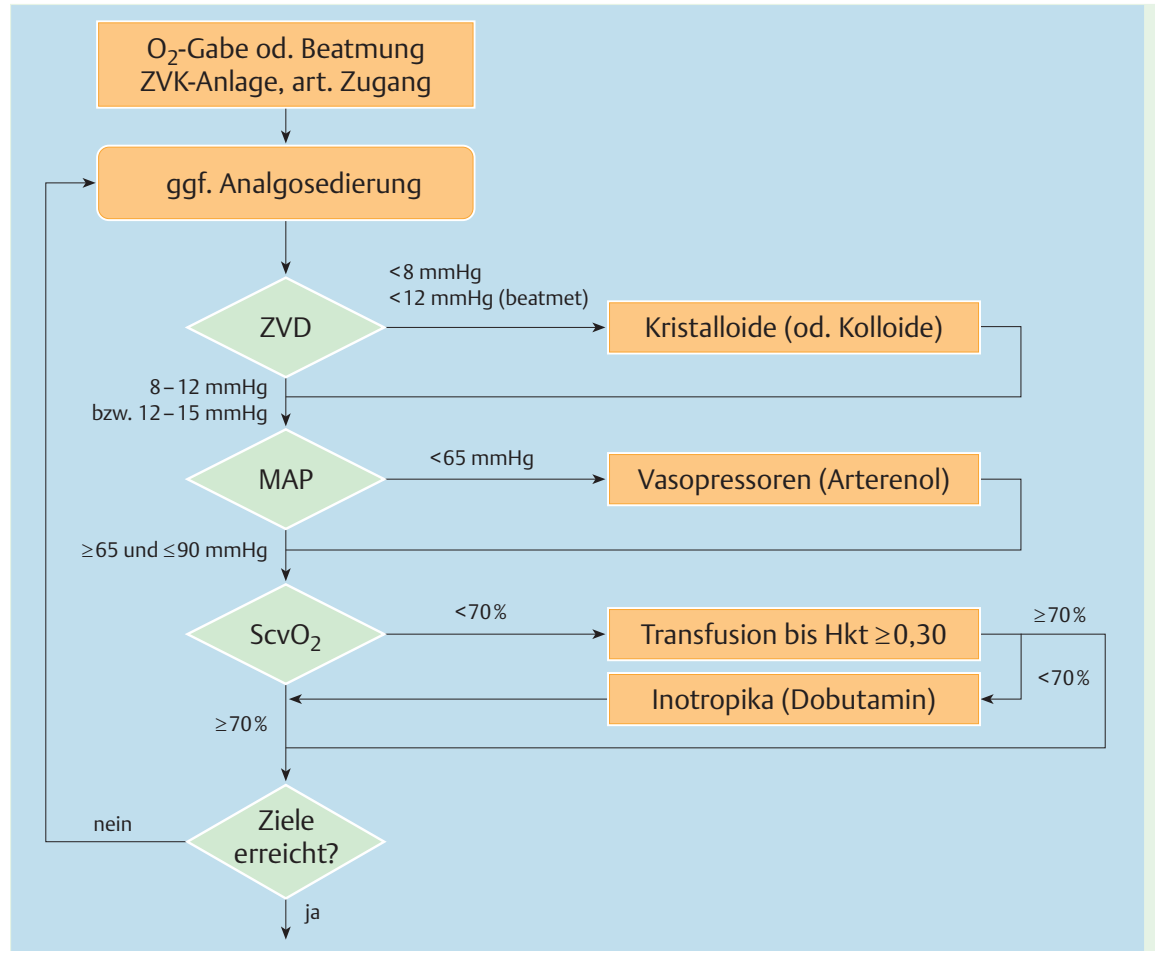

Abb. 3 Algorithmus der „early goal directed therapy“ des septischen Schocks nach Rivers u. Mitarb. [39].

Hkt: Hämatokrit

MAP: Mittlerer arterieller Druck

$\mathrm{ScvO}_{2}$ : Zentralvenöse Sauerstoffsättigung

ZVD: Zentralvenöser Druck

nisse verdoppelte und die Gesamtprognose (90-Tage-Letalität) tendenziell beeinträchtigte [44].

Die Vasopressorentherapie zielt auf die Anhebung des mittleren arteriellen Blutdruckes auf über $60 \mathrm{mmHg}$, der unteren Grenze der Zone der Autoregulation des Gefäßbettes in den Zielorganen Gehirn, Koronarien und Niere. Dies kann aber potenziell mit einer peripheren Ischämie infolge Gefäßkonstriktion erkauft werden. Vor allem Dopamin, Noradrenalin und Vasopressin wurden in den letzten Jahren geprüft. Dopamin zeichnet sich zwar durch eine potente Vasokonstriktion, aber auch durch eine positiv chronotrope und proarrhythmogene Wirkung aus. Es erhöht den pulmonal arteriellen Verschlussdruck und die pulmonale Shuntfraktion und wirkt über eine Depression der Prolaktin-Sekretion immunsupprimierend [43]. In Deutschland wird aus diesen Gründen vor allem Noradrenalin eingesetzt. Eine Untersuchung von Noradrenalin gegenüber Vasopressin (in Deutschland nicht zugelassen) erfolgte 2008 durch eine Studie von Russell u. Mitarb. [45]. Hier hatte die niedrig dosierte Dauerinfusion von Vasopressin zusätzlich zu Noradrenalin keinen Effekt auf das Behandlungsergebnis.

\section{Maschinelle Beatmung}

In 30 bis $60 \%$ der Fälle geht ein septischer Schock mit einem akuten Lungenversagen (ARDS) einher. Das ARDS tritt bei pulmogener Sepsis infolge einer Pneumonie besonders häufig auf. Die akute Schädigung des respiratorischen Epithels ist entzündlichtoxischer Natur. Sie wird von einem proteinreichen, normotonen Lungenödem begleitet. Die maschinelle Beatmung wird zur Therapie der dabei auftretenden schweren, auf Sauerstoffgabe refraktären Hypoxämie notwendig. Außerdem kann der Patient die erforderliche, stark erhöhte Atemarbeit oft nicht mehr aufbringen. Nicht vermeidbare Folgen der maschinellen Atemhilfe sind positive intrapulmonale Drücke, atemzyklusabhängige Druckschwankungen und aufgrund der hohen Öffnungsdrucke des geschädigten Lungenparenchyms erhebliche Gewebescher- kräfte mit zyklischer, traumatischer Öffnung atelektatischer Lungenareale, die die alveolo-kapilläre Einheit mechanisch weiter schädigen und durch Induktion proinflammatorischer Mediatoren und Mobilisierung mikrobieller Pathogene den Entzündungsprozess lokal und systemisch perpetuieren [46-49].

In einer Serie von Studien hat das nordamerikanische ARDS-Network, ausgehend von früheren Untersuchungen von Amato u. Mitarb. [50] ein Konzept entwickelt, mit dem der pulmonale Gasaustausch unterstützt werden kann und die Ventilator-assoziierte Lungenschädigung zugleich minimiert wird. Dieses Konzept ist als „protective ventilation strategy“ bekannt geworden. Es besteht aus folgenden Komponenten:

Aufrechterhaltung einer ausreichenden arteriellen Sauerstoffsättigung. Sie beträgt bei erhöhtem Sauerstoffbedarf in der Sepsis $\geq 95 \%$, ansonsten ist eine Sättigung von 88-90\% ausreichend. Die inspiratorische Sauerstoffkonzentration $\left(\mathrm{F}_{\mathrm{i}} \mathrm{O}_{2}\right)$ wird diesem Bedarf angepasst. Bei einem $\mathrm{F}_{\mathrm{i}} \mathrm{O}_{2}$ von $\geq 0,6$ besteht die Gefahr toxischer Sauerstoffeffekte. Er soll möglichst nicht überschritten werden.

Bei Druck- oder Volumen-kontrolliertem Beatmungsmodus wird das Atemzugvolumen auf $6 \mathrm{ml} / \mathrm{kg}$ Sollkörpergewicht begrenzt [51]. Der obere Beatmungsdruckes wird auf $30 \mathrm{~cm} \mathrm{H}_{2} \mathrm{O}$ begrenzt und die weitere Absenkung des Inspirationsdruckes auf unter $30 \mathrm{~cm} \mathrm{H}_{2} \mathrm{O}$ genießt hohe Priorität, da es keine sichere Obergrenze gibt [52]. Zur Gewährleistung einer ausreichenden alveolären Ventilation wird die Atemfrequenz erhöht (in der Regel auf über 20/min). Eine respiratorische Azidose wird bis zu einem pH-Wert von 7,2 zugelassen [53].

Zur Eröffnung und permanenten Aufrechterhaltung einer möglichst großen Gasaustauschfläche und zur Vermeidung von Gewebescherkräften („Atelektrauma“) wird maschinenseitig ein erhöhter positiver endexspiratorischer Druck (PEEP) gewählt. Die Ermittlung des optimalen PEEP kann auf mehreren Wegen erfolgen: Durch ein Protokoll, bei dem die PEEP-Höhe von der Schwere der Gasaustauschstörung abhängig gemacht wird [51,54] oder durch Beurteilung der Volumenrekrutierung/Gasaustauschver- 
besserung unter einer Titration des PEEP nach oben. Die Frage, ob grundsätzlich ein hoher PEEP ( $>12 \mathrm{~cm} \mathrm{H}_{2} \mathrm{O}$ ) oder ein mäßiger PEEP $\left(\sim 8-12 \mathrm{~cm} \mathrm{H}_{2} \mathrm{O}\right)$ prognostisch günstiger ist, konnte in drei aktuellen prospektiven, randomisierten Studien nicht geklärt werden [54-56]. In allen Untersuchungen war die Letalität in der High-PEEP-Gruppe jedoch tendenziell - aber nicht statistisch signifikant - niedriger. Ein wichtiger Befund ist jedoch, dass bei Patienten mit primär pulmonaler Schädigung (pulmogene Sepsis im Rahmen einer schweren Pneumonie) häufig durch sukzessive PEEP-Erhöhung über mäßige Drücke hinaus nur eine geringe oder keine Rekrutierung der Gasaustauschfläche gelingt [57]. Morphologisch liegt dabei oft nach computertomografischen Kriterien ein fokal verteiltes ARDS vor. Dann überwiegt bei hohem PEEP die Gefahr der Lungenüberdehnung gegenüber der Chance der Lungenrekrutierung. Die Anpassung des PEEP-Niveaus sollte daher individuell nach vorgewählten physiologischen Zielen (Anstieg des Atemzugvolumens oder der arteriellen Sauerstoffsättigung im Rahmen der PEEP-Titration) erfolgen [57].

Andere Aspekte der maschinellen Beatmung bei ARDS (z. B. die sogenannte kinetische Therapie, extrakorporale Gasaustauschverfahren) führen in Zusammenhang mit der Betrachtung der Sepsis zu weit. Es sei auf die weiterführende Literatur verwiesen [58].

\section{Korrektur der endokrinen Deregulation \\ $\nabla$}

Etwa jeder zweite Patient im septischen Schock weist eine relative adrenale Insuffizienz auf $[59,60]$. Sie ist definiert durch einen Anstieg des Serum-Cortisols um weniger als $9 \mu \mathrm{g} / \mathrm{dl}$ nach Stimulation mit $250 \mu \mathrm{g}$ synthetischem Corticotropin [61]. Etwa 10\% der Patienten haben eine absolute Nebenniereninsuffizienz [61]. Diese Störungen sind mit einer stark erhöhten 28-Tage-Letalität assoziiert [59,60,62]. Nachdem Studien mit pharmakodynamischen Kortikosteroiddosen erfolglos blieben, zeigte eine post hoc Analyse einer prospektiven, randomisierten, kontrollierten Studie mit niedrig dosiertem Hydrocortison $(200 \mathrm{mg} / \mathrm{Tag} \times 5$ Tage, Ausschleichen über 6 Tage) eine Prognoseverbesserung bei Patienten mit relativer Nebenniereninsuffizienz an [63]. In der Intention-to-treat-Analyse der Gesamtpopulation fand sich kein Unterschied in der Gesamtletalität. Die aktuell publizierte, europäische CORTICUS-Studie untersuchte 499 Patienten im gleichen Design und stratifizierte zwischen Patienten mit erhaltener Nebennierenfunktion und solchen mit relativer adrenaler Insuffizienz [60]. Auch hier bestätigte sich die um etwa $10 \%$ höhere Letalität der letzteren Patientengruppe, aber es fand sich kein Unterschied in der Letalität der Verum- gegenüber der Placebogruppe, unabhängig vom Funktionszustand der Nebenniere. Die Rückbildung des Schocks gelang schneller in der Verumgruppe. Es gab dort aber eine höhere Rate an Superinfektionen, einschließlich Sepsis und septischem Schock. Damit kann gegenwärtig keine Empfehlung für eine Kortikosteroidtherapie bei schwerer Sepsis ausgesprochen werden, es sei denn, es liegt eine absolute Nebenniereninsuffizienz vor. Dies sollte bei persistenter arterieller Hypotonie trotz regelrechter Therapie geprüft werden (basaler Cortisolspiegel von unter $10 \mu \mathrm{g} / \mathrm{dl}$ Plasma).

Ähnlich ernüchternde Daten liegen zur Normalisierung des Blutzuckers mittels intensiver Insulintherapie vor. In einem Kollektiv von kritisch kranken, maschinell beatmeten Patienten einer chirurgischen Intensivstation berichteten Van den Berghe u. Mitarb. [64] über eine Erniedrigung der Gesamtletalität um etwa 30\% durch Normalisierung des Blutzuckers (Ziel: 80 - $110 \mathrm{mg} / \mathrm{dl}$ ) mittels einer intensiven Insulintherapie. Am meisten profitierten kardiochirurgische Patienten mit schwerer Sepsis und Multiorganversagen nach massiver perioperativer Infusion von Glukose. Obwohl eine Nachfolgestudie der gleichen Arbeitsgruppe bei Intensivpatienten aus dem nicht-operativen Bereich den günstigen Effekt nicht bestätigen konnte [65], wurde die intensive Insulintherapie in vielen Intensivstationen adoptiert. Eine große, multizentrische Studie zur Prüfung des gleichen Blutglukose-Zielwertes im Normalbereich aus dem Jahr 2008 [44] fand keine Verbesserung der Prognose gegenüber einer konventionellen Insulintherapie (Zielwert der Blutglukose: $180-200 \mathrm{mg} / \mathrm{dl}$ ), sondern eine erhöhte Rate schwerer Hypoglykämien $(<40 \mathrm{mg} / \mathrm{dl})$ von $17 \%$ gegenüber $4 \%$.

\section{Interventionen im Gerinnungssystem \\ $\nabla$}

Die Sepsis-assoziierte Entzündungskaskade führt zur Gerinnungsaktivierung und zur Inhibition der Fibrinolyse. Die resultierende Thrombinbildung fördert nicht nur Fibrinaggregate im Kapillarbett („disseminerte intravasale Gerinnung“), sondern unterhält über direkte und indirekte Mechanismen die systemische Inflammation [66]. Das endogene Antikoagulans Antithrombin III (ATIII) inhibiert die prokoagulatorischen und proinflammatorischen Prozesse $[67,68]$. Es fällt in der schweren Sepsis dramatisch ab und dieser Vorgang korreliert mit dem Schweregrad der Erkrankung. Die Ersatztherapie mit exogenem, hochdosiertem ATIII bei schwerer Sepsis wurde in mehreren Placebo-kontrollierten Studien prospektiv geprüft. In der größten dieser Studien (Kybersept) konnte kein Unterschied in der 28-Tage-Letalität gegen Placebo gefunden werden (38,9\% vs. 38,7\%) [69]. Auch eine aktuelle Metaanalyse fand keine Erniedrigung des Letalitätsrisikos durch Hochdosis-ATIII, auch nicht in Subgruppenanalysen [70]. Dem gegenüber kamen die Kybersept-Untersucher in einer post hoc Analyse der Patienten mit hohem Letalitätsrisiko (prospektive Letalität 30-60\% nach dem SAPS II Score) zu einem positiven Ergebnis: Die 28-Tage-Letalität betrug unter ATIII 35,7\% versus $44,4 \%$ in der Placebogruppe [71]. ATIII wird gegenwärtig nicht in der Sepsistherapie empfohlen [41].

2001 wurde rekombinantes aktiviertes Protein C (Drotregocin [aktiviert], DrotAA) als erstes spezifisches Medikament zur Therapie der Sepsis zugelassen. Dies erfolgte aufgrund der Ergebnisse einer großen Phase III-Prüfung, die eine Senkung der Letalität von $30,8 \%$ in der Placebogruppe versus $24,7 \%$ in der Studienpopulation ergab [72]. Protein C ist ein Proenzym, das von Thrombin im Komplex mit Thrombomodulin zu aktivertem Protein C umgewandelt wird. Dieses inaktiviert zusammen mit Protein S Gerinnungsfaktoren und wirkt auf diese Weise antithrombotisch. Bei schwerer Sepsis ist Protein C im Plasma vermindert und eine erniedrigte Protein C-Plasmakonzentrationen zeigt eine erhöhte Letalität an. DrotAA wurde nur bei schwerer Sepsis geprüft. Die Wirkung war von der Protein C-Plasmakonzentration unabhängig; sie war am ausgeprägtesten bei pulmogener Sepsis, war aber dagegen bei Urosepsis nicht nachweisbar. Patienten mit Mehrorganversagen, und solche, bei denen die Therapie innerhalb der ersten 24 Stunden der Organdysfunktion begonnen wurde, profitierten überdurchschnittlich gut $[73,74]$. Patienten mit niedrigem Letalitätsrisiko (Acute Physiology and Chronic Health Evaluation [APACHE]-Score < 25) haben keinen Nutzen von der Therapie [75]. Das Blutungsrisiko ist unter DrotAA klinisch signifikant erhöht und ein hohes Blutungsrisiko stellt eine Kontraindikation für seinen Einsatz dar. Eine begleitende prophylaktische Gabe von Heparin ist bei niedrigem Blutungsrisiko nicht kontraindiziert [76]. 


\section{Schlussfolgerungen}

Nach den Kriterien der Evidenz-basierten Medizin sind viele der in der Intensivmedizin praktizierten Diagnose- und Therapieverfahren nicht zweifelsfrei gesichert. Die rationale Therapie der Sepsis ist jedoch aufgrund ihrer hohen Prävalenz und der hohen Letalität ein vordringliches Ziel. Mit der Durchführung großer kontrollierter, multizentrischer Studien hat sich die empirische Basis der Sepsistherapie in den letzten 10 Jahren erheblich verbessert. Grundlage dafür waren die Bildung von Kompetenznetzwerken wie die „Surviving Sepsis Campaign“ und das „SepNet“, aber auch Initiativen der pharmazeutischen Industrie. Diese Entwicklung wird schon in den nächsten Jahren weitere Früchte tragen. Der Einsatz der hier entwickelten Therapiekomponenten darf allerdings nie schematisch und muss immer unter Berücksichtigung der Gesamtsituation des Patienten erfolgen. Beispielsweise ist die aggressive Volumentherapie des septischen Schocks dann zu begrenzen, wenn ein schweres Lungenversagen im Vordergrund steht, da die Prognose des Lungenversagens durch Flüssigkeitsüberladung negativ beeinflusst wird [77].

Es wird immer deutlicher, dass ähnlich wie bei akuten Gefäßereignissen in der zerebralen und kardialen Strombahn, die schwere Sepsis einen Notfall darstellt, der innerhalb einer Stunde Maßnahmen in geordneter Abfolge erfordert und deren Verzögerung oder Unterlassung die Prognose des Patienten erheblich verschlechtert. Dazu gehören zum Beispiel die empirische Initialbehandlung mit Antiinfektiva und die Schocktherapie. Die zeitgerechte Bearbeitung eines entsprechenden Algorithmus setzt eine enge Verzahnung von Notfallmedizin und Intensivmedizin voraus [40]. Sowohl die Verbesserung des therapeutischen Armamentariums wie die Optimierung der Abläufe im Patientenmanagement werden zu einer weiteren Verbesserung der Prognose der schweren Sepsis beitragen.

\section{Danksagung}

Die vorliegende Arbeit widme ich den Mitarbeitern der internistischen Intensivmedizin meiner Klinik. Ich bedanke mich damit für den kontinuierlichen gemeinsamen Prozess zur Optimierung unserer Patientenbetreuung.

\section{Literatur}

1 Bone RC, Balk RA, Cerra FB et al. Definitions for sepsis and organ failure and guidelines for the use of innovative therapies in sepsis. The ACCP/ SCCM Consensus Conference Committee. Chest 1992; 101: 16441655

2 Vincent JL. Dear SIRS, I'm sorry to say that I don't like you. Crit Care Med 1997; 25: 372 - 374

3 Alberti C, Brun-Buisson C, Chevret $S$ et al. Systemic inflammatory response and progression to severe sepsis in critically ill infected patients. Am J Respir Crit Care 2005; 171: 461 - 468

4 Wheeler AP, Bernard GR. Treating patients with severe sepsis. N Engl J Med 1999; 340: 207-214

5 Annane D, Bellisant E, Cavaillon JM. Septic shock. Lancet 2005; 365: $63-78$

6 Sands KE, Bates DW, Lanken PN et al. Epidemiology of sepsis syndrome in 8 academic medical centers. JAMA 1997; 278: 234-240

7 Angus D, Linde-Zwirble WT, Lidicker J et al. Epidemiology of severe sepsis in the United States: analysis of incidence, outcome, and associated cost of care. Crit Care Med 2001; 29: 1303 - 1310

8 Annane D, Aegerter P, Jars-Guincestre MC et al. Current epidemiology of septic shock. Am J Respir Crit Care Med 2003; 168: 165-172

9 Brunkhorst FM, Engel C, Welte T et al. Prevalence of infection in German Intensive Care Units - Results from the German Prevalence Study. Infection 2005; 33 (Suppl 1): 47
10 Marini JJ, Wheeler AP. Severe Sepsis. In: Marini JJ, Wheeler AP (eds). Critical Care Medicine - The Essentials, 3rd Edition. Philadelphia: Lippincott, Williams and Wilkins, 1997: 451 - 465

11 Wurfel MM, Gordon AC, Holden TD et al. Toll-like receptor 1 polymorphisms affect innate immune responses and outcome in sepsis. Am J Respit Crit Care Med 2008; 178: 710 - 720

12 Christ-Crain M, Stolz D, Bingisser R. Effect of procalcitonin-guided therapy in community-acquired pneumonia: a randomized trial. Am J Respir Crit Care Med 2006; 174: 84-93

13 Harbarth S, Holeckova K, Froidevaux C. Diagnostic value of procalcitonin, interleukin-6, and interleukin-8 in critically ill patients admitted with suspected sepsis. Am J Respir Crit Care Med 2001; 164: 396- 402

14 Nobre V, Harbarth S, Graf JD et al. Use of procalcitonin to shorten antibiotic treatment duration in septic patients. Am J Respir Crit Care Med 2008; 177: 498 - 505

15 Brunkhorst FM, Engel C, Jaschinsky U et al. Treatment of severe sepsis and septic shock in Germany - the gap between perception and practice. Results from the German prevalence study. Intensivmed.up2date 2006; $2: 89-90$

16 Intravenous Immunoglobulin Collaborative Study Group. Prophylactic intravenous administration of standard immune globulin as compared with core-lipopolysaccharide immune globulin in patients at high risk of postsurgical infection. N Engl J Med 1992; 327: 234-240

17 Cohen J, Carlet J. INTERSEPT: an international, multicenter, placebocontrolled trial of monoclonal antibody to human tumor necrosis factor-alpha in patients with sepsis. International Sepsis Trial Study Group. Crit Care Med 1996; 24: 1431 - 1440

18 Panacek EA, Marshall J, Fischkoff S et al. Neutralization of TNF by a monoclonal antibody improves survival and reduces organ dysfunction in human sepsis: results of the MONARCS trial. Chest 2000; 118 (Suppl): 88

19 Abraham E, Reinhart K, Opal S, Demeyer I, Doig C for the OPTIMIST Trial Study Group. Efficacy and safety of Tifacogin (recombinant tissue factor) in severe sepsis. JAMA 2003; 290: 238-247

20 Angstwurm MWA, Engelmann L, Zimmermann T et al. Selenium in intensive care (SIC): Results of a prospective randomized, placebo-controlled, multiple center study in patients with severe systemic inflammatory response syndrome, sepsis, and septic shock. Crit Care Med 2007; 325: $118-126$

21 Kumar A, Roberts D, Wood KE et al. Duration of hypotension before initiation of effective antimicrobial therapy is the critical determinant of survival in human septic shock. Crit Care Med 2006; 34: 1589-1595

22 Garey KW, Rege M, Pai MP et al. Time to initiation of fluconazole therapy impacts mortality in patients with candidemia: A multi-institutional study. Clin Infect Dis 2006; 43: 25-31

23 McGarvey RN, Harper JJ. Pneumonia mortality reduction and quality improvement in a community hospital. QRB Qual Rev Bull 1993; 19: $124-130$

24 Meehan TP, Fine MJ, Krumholz HM. Quality of care, process, and outcomes in elderly patients with pneumonia. JAMA 1997; 278: 20802084

25 Iregui M, Ward S, Sherman G. Clinical importance of delays in the initiation of appropriate antibiotic treatment for ventilator-associated pneumonia. Chest 2002; 122: 262-268

26 Gacouin A, Le Tulco Y, Lavoue S. Severe pneumonia due to Legionella pneumophila: Prognostic factors, impact of delayed appropriate antimicrobial therapy. Intensive Care Med 2002; 28: 686-691

27 Proulx N, Frechette D, Toye B. Delays in the administration of antibiotics are associated with mortality from adult acute bacterial meningitis. QJM 2005; 98: 291-298

28 Kang CI, Kim SH, Kim HB. Pseudomonas aeruginosa bacteremia: Risk factors for mortality and influence of delayed receipt of effective antimicrobial therapy on clinical outcome. Clin Infect Dis 2003; 37: $745-$ 751

29 Lodise TP, McKinnon PS, Swiderski L. Outcome analysis of delayed antibiotic treatment for hospital-acquired Staphylococcus aureus bacteremia. Clin Infect Dis 2003; 36: 1418-1423

30 Höffken G, Lorenz J, Kern WV et al. S3-Leitlinie der Paul-Ehrlich-Gesellschaft für Chemotherapie, der Deutschen Gesellschaft für Pneumologie, der Deutschen Gesellschaft für Infektiologie und des Kompetenznetzwerkes CAPNETZ zu Epidemiologie, Diagnostik, antimikrobieller Therapie und Management von erwachsenen Patienten mit ambulant erworbenen tiefen Atemwegsinfektionen (akute Bronchitis, akute Exazerbation einer chronischen Bronchitis, Influenza und andere respira- 
torische Virusinfektionen) sowie ambulant erworbener Pneumonie. Pneumologie 2005; 59: 612-664

31 Gleason PP, Meehan TP, Fine JM et al. Associations between initial antimicrobial therapy and medical outcomes for hospitalized elderly patients with pneumonia. Arch Intern Med 1999; 159: 2562 - 2572

32 Houck PM, MacLehose RF, Niederman MS et al. Empiric antibiotic therapy and mortality among medicare pneumonia inpatients in 10 western states: 1993, 1995, and 1997. Chest 2001; 119: 1420-1426

33 Martinez JA, Horcajada JP, Almela $M$ et al. Addition of a macrolide to a beta-lactam-based empirical antibiotic regimen is associated with lower in-hospital mortality for patients with bacteremic pneumococcal pneumonia. Clin Infect Dis 2003; 36: 389- 395

34 Mufson MA, Stanek RJ. Bacteremic pneumococcal pneumonia in one American City: a 20-year longitudinal study, 1978-1997. Am J Med 1999; 107: 34S - 43S

35 Stahl JE, Barza M, DesJardin J et al. Effect of macrolides as part of initial empiric therapy on length of stay in patients hospitalized with community-acquired pneumonia. Arch Intern Med 1999; 159: 2576-2580

36 Baddour LM, Yu VL, Klugman KP et al. Combination antibiotic therapy lowers mortality among severely ill patients with pneumococcal bacteremia. Am J Respir Crit Care Med 2004; 170: 440 - 444

37 Chastre J, Wolff M, Fagon JY et al. Comparison of 8 vs 15 days of antibiotic therapy for ventilator-associated pneumonia in adults: a randomized trial. JAMA 2003; 290: 2588-2598

38 Rodriguez A, Mendia A, Sirvent JM et al. Combination antibiotic therapy improves survival in patients with community-acquired pneumonia and shock. Crit Care Med 2007; 35: 1493 - 1498

39 Rivers E, Nguyen B, Havstad S. Early goal directed therapy in the treatment of severe sepsis and septic shock. N Engl J Med 2001; 345: 1368 1377

40 Trzeciak S, Dellinger RP, Abate NL. Translating research to clinical practice: a 1-year experience with implementing early goal-directed therapy for septic shock in the emergency department. Chest 2006; 129 : $225-232$

41 Dellinger RP, Levy MM, Carlet JM et al. Surviving Sepsis Campaign: International guidelines management of severe sepsis and septic shock: 2008. Critical Care Med 2008; 36: 296 - 327

42 Wilkes MM, Navickis RJ. Patient survival after human albumin administration. A meta-analysis of randomized, controlled trials. Ann Intern Med 2001; 135: 149-164

43 Hollenberg SM, Ahrens TS, Annane D et al. Practice parameters for hemodynamic support of sepsis in adult patients: 2004 update. Crit Care Med 2004; 32: $1928-1948$

44 Brunkhorst FM, Engel C, Bloos F et al. Intensive insulin therapy and pentastarch rescucitation in severe sepsis. N Engl J Med 2008; 358: 125 139

45 Russell JA, Walley KR, Singer J et al. Vasopressin versus norepinephrin infusion in patients with septic shock. N Engl J Med 2008; 358: 877 887

46 Charles PE, Martin L, Etienne $M$ et al. Influence of positive end-expiratory pressure (PEEP) on histopathological and bacteriological aspects of pneumonia during low tidal volume mechanical ventilation. Intensive Care Med 2004; 30: 2263 - 2270

47 Sakr Y, Vincent JL, Reinhart Ket al. High tidal volume and positive fluid balance are associated with worse outcome in acute lung injury. Chest 2005; 128: 3098 - 3018

48 Stüber F, Wrigge H, Schröder S et al. Kinetic and reversibility of mechanical ventilation-associated pulmonary and systemic inflammatory response in patients with acute lung injury. Intensive Care Med 2002; 28: $834-841$

49 Dreyfuss $D$, Saumon $S$. State of the art: Ventilator-induced lung injury. Am J Respir Crit Care Med 1998; 157: 294-323

50 Amato MB, Barbas CS, Medeiros DM et al. Effect of a protective-ventilation strategy on mortality in the acute respiratory distress syndrome. N Engl J Med 1997; 338: 347 - 354

51 The Acute Respiratory Distress Syndrome Network. Ventilation with lower tidal volumes as compared with traditional tidal volumes for acute lung injury and the acute respiratory distress syndrome. N Engl J Med 2000; 342: 1301 - 1308

52 Hager DN, Krishnan JA, Hayden DL et al. Tidal volume reduction in patients with acute lung injury when plateau pressures are not high. Am J Respir Crit Care Med 2005; 172: 1241 - 1245

53 Hickling $K G$, Henderson SJ, Jackson L. Low mortality associated with low volume pressure limited ventilation with permissive hypercapnia in severe adult respiratory distress syndrome. Intensive Care Med 1990; 16: $372-377$

54 Brower RG, Lanken PN, MacIntyre N. Higher versus lower positive endexpiratory pressures in patients with the acute respiratory distress syndrome. N Engl J Med 2004; 351: 327 - 336

55 Mercat A, Richard JM, Vielle B. Positive end-expiratory pressure setting in adults with acute lung injury and acute respiratory distress syndrome (EXPRESS). JAMA 2008; 299: 646-655

56 Meade MO, Cook DJ, Guyatt GH. Ventilation strategy using low tidal volumes, recruitment maneuvers, and high positive end-expiratory pressure for acute lung injury and acute respiratory distress syndrome: a randomized controlled trial. JAMA 2008; 299: 637-645

57 Grasso S, Stripoli T, De Michele $M$ et al. ARDSnet Ventilatory Protoco and Alveolar Hyperinflation: Role of Positive End-Expiratory Pressure. Am J Respir Crit Care Med 2007; 176: 761 - 767

58 Lorenz J. Akuter Lungenschaden und akutes Atemnotsyndrom (ARDS). In: Weilemann S, Lorenz J, Voigtländer T (Hrsg). Internistische Intensivmedizin und Notfallmedizin. Heidelberg: Springer, 2007: 249-260

59 Rothwell PM, Udwadia ZF, Lawler PG. Cortisol response to corticotropin and survival in sepsis. Lancet 1991; 337: $582-583$

60 Sprung CL, Annane D, Keh D et al. Hydrocortisone therapy for patients with septic shock. N Engl J Med 2008; 358: 111 - 124

61 Annane D, Maxime V, Ibrahim F et al. Diagnosis of adrenal insufficiency in severe sepsis and septic shock. Am J Respir Crit Care Med 2006; 174 : $1319-1326$

62 Annane D, Sébille V, Troché G et al. A 3-level prognostic classification in septic shock based on cortisol levels and cortisol response to corticotropin. JAMA 2000; 283: 1038-1045

63 Annane D, Sebille V, Charpentier C et al. Effect of treatment with low doses of hydrocortisone and fludrocortisones on mortality in patients with septic shock. JAMA 2002; 288: 862 - 871

64 Van den Berghe $G$, Wouters P, Weekers F. Intensive insulin therapy in critically ill patients. N Engl J Med 2001; 345: 1359-1367

65 Van den Berghe $G$, Wilmer A, Hermans G. Intensive insulin therapy in the medical ICU. N Engl J Med 2006; 354: 449 - 461

66 Levi $M$, de Jonge $E$, van der Poll T. New treatment strategies for disseminated intravascular coagulation based on current understanding of the pathophysiology. Ann Med 2004; 36: 41 - 49

67 Opal SM, Kessler CM, Roemisch J et al. Antithrombin, heparin, and heparan sulfate. Crit Care Med 2002; 30 (5 suppl): 325 - 331

68 Rublee D, Opal SM, Schramm Wet al. Quality of life effects of antithrombin III in sepsis survivors: results from the KyberSept trial. Crit Care 2002; 6: 349-356

69 Warren BL, Eid A, Singer P et al. KyberSept Trial Study Group: High-dose antithrombin III in severe sepsis. A randomized controlled trial. JAMA 2001; 286: $1869-1878$

70 Afshari A. Antithrombin III in critically ill patients: Systematic review with meta-analysis and trial sequential analysis. BMJ 2007; 335: 248- 1256

71 Wiedermann CJ, Hoffmann JN, Juers $M$ et al. High-dose antithrombin II in the treatment of severe sewpsis in patients with a high risk of death: Efficacy and safety. Crit Care Med 2006; 34: 285-291

72 Bernard GR, Vincent JL, Laterre PF et al. For the recombinant human activated protein $C$ worldwide evaluation in severe sepsis (PROWESS) study group: efficacy and safety of recombinant human activated protein C for severe sepsis. N Engl J Med 2001; 344: 699-709

73 Dhainaut JF, Laterre PF, Janes JM et al. Drotrecogin alfa (activated) in the treatment of severe sepsis patients with multiple-organ failure dysfunction: data from the PROWESS trial. Intensive Care Med 2003; 29: $894-903$

74 Vincent JL, Bernard GR, Beale $R$ et al. Drotrecogin alfa (activated) treatment in severe sepsis from the global open-label trial ENHANCE: Further evidence for survival and safety and implications for early treatment. Crit Care Med 2005; 33: 2266-2277

75 Abraham E, Laterre PF, Talwar D et al. Drotrecogin alfa (activated) for adults with severe sepsis and a low risk of death. N Engl J Med 2005; 353: $1332-1341$

76 Levi M, Levy M, Williams MD et al. Prophylactic heparin in patients with severe sepsis treated with drotrecogin alfa (activated). Am J Respir Crit Care Med 2007; 176: 483-490

77 The NHLBI ARDS Network. Comparison of two fluid-management strategies in acute lung Injury. N Engl J Med 2006; 354: 2564 - 2575 